\title{
Pacific
}

Journal of

Mathematics

\section{WEAKLY KRULL DOMAINS AND THE COMPOSITE NUMERICAL SEMIGROUP RING $D+E\left[\Gamma^{*}\right]$}

JUNG WOOK LIM 


\title{
WEAKLY KRULL DOMAINS AND THE COMPOSITE NUMERICAL SEMIGROUP RING $D+E\left[\Gamma^{*}\right]$
}

\author{
JUNG WOOK LIM
}

\begin{abstract}
Let $D \subseteq E$ be an extension of integral domains, $\Gamma$ a numerical semigroup with $\Gamma \subsetneq \mathbb{N}_{0}, \Gamma^{*}=\Gamma \backslash\{0\}$ and $R=D+E\left[\Gamma^{*}\right]$. In this paper, we completely characterize when $R$ is a weakly Krull domain, an AWFD or a GWFD. We also prove that $R$ is never a WFD.
\end{abstract}

\section{Introduction}

We first review some preliminaries. Let $D$ be an integral domain with quotient field $q f(D)$ and let $\mathbf{F}(D)$ denote the set of nonzero fractional ideals of $D$. Recall that the $v$-operation on $D$ is a star-operation on $\mathbf{F}(D)$ defined by $I \mapsto I_{v}:=\left(I^{-1}\right)^{-1}$, where $I^{-1}=\{x \in q f(D) \mid x I \subseteq D\}$. The $t$-operation on $D$ is a star-operation defined by $I \mapsto I_{t}:=\bigcup\left\{J_{v} \mid J \subseteq I\right.$ with $J \in \mathbf{F}(D)$ finitely generated $\}$. An $I \in \mathbf{F}(D)$ is said to be a $v$-ideal if $I_{v}=I$, and a $t$-ideal if $I_{t}=I$. A $v$-ideal $I$ is said to be of finite type if $I=J_{v}$ for some finitely generated fractional ideal $J$ of $D$. A $t$-ideal $M$ of $D$ is called a maximal $t$-ideal if $M$ is maximal among proper integral $t$-ideals of $D$. It is well known that maximal $t$-ideals are prime ideals. Let $t-\operatorname{Max}(D)$ be the set of maximal $t$-ideals of $D$. Then $t-\operatorname{Max}(D) \neq \varnothing$ if $D$ is not a field. An $I \in \mathbf{F}(D)$ is said to be $t$-invertible if $\left(I I^{-1}\right)_{t}=D$; equivalently, $I I^{-1} \nsubseteq M$ for each $M \in t$-Max $(D)$. Let $T(D)$ be the abelian group of $t$-invertible fractional $t$-ideals of $D$ under the $t$ multiplication $I * J=(I J)_{t}$, and let $\operatorname{Inv}(D)$ and $\operatorname{Prin}(D)$ be the subgroups of $T(D)$ consisting respectively of invertible fractional ideals of $D$ and nonzero principal fractional ideals of $D$. Then it is clear that $\operatorname{Prin}(D) \subseteq \operatorname{Inv}(D) \subseteq T(D)$. The $t$-class group of $D$ is an abelian group $\mathrm{Cl}(D)=T(D) / \operatorname{Prin}(D)$ and the Picard group $\operatorname{Pic}(D)=\operatorname{Inv}(D) / \operatorname{Prin}(D)$ is a subgroup of $\mathrm{Cl}(D)$. The local t-class group $G(D)$ of $D$ is defined by $G(D)=\mathrm{Cl}(D) / \operatorname{Pic}(D)$.

Let $X^{1}(D)$ stand for the set of height-one prime ideals of $D$. We say that $D$ is a weakly Krull domain if $D=\bigcap_{P \in X^{1}(D)} D_{P}$ and this intersection has finite character, i.e., each nonzero element $d \in D$ is a unit in $D_{P}$ for all but a finite number of $P$ 's in $X^{1}(D) ; D$ is a weakly factorial domain (WFD) if every nonzero nonunit element of $D$ is a product of primary elements; $D$ is an almost weakly factorial domain

MSC2010: primary 13A15, 13G05; secondary 13A02, 13B25, $13 \mathrm{~F} 05$.

Keywords: numerical semigroup, $D+E\left[\Gamma^{*}\right]$, weakly Krull domain. 
(AWFD) if for each nonzero nonunit element $d \in D$, there exists a positive integer $n=n(d)$ such that $d^{n}$ is a product of primary elements; and $D$ is a generalized weakly factorial domain (GWFD) if each nonzero prime ideal of $D$ contains a primary element. (Recall that a nonzero nonunit $d \in D$ is called a primary element of $D$ if $(d)$ is a primary ideal of $D$.) It is well known that

\section{$\mathrm{WFD} \Rightarrow \mathrm{AWFD} \Rightarrow$ GWFD $\Rightarrow$ weakly Krull domain}

and a weakly Krull domain has $t$-dimension one. (The $t$-dimension of $D$, abbreviated $t-\operatorname{dim}(D)$, is the supremum of lengths of chains of prime $t$-ideals of $D$. Hence $t-\operatorname{dim}(D)=1$ if and only if each maximal $t$-ideal of $D$ has height-one.) Also, it was shown in [Anderson and Zafrullah 1990, Theorem] that a weakly Krull domain $D$ is a WFD if and only if $\mathrm{Cl}(D)=0$, and in [Anderson et al. 1992, Theorem 3.4] that a weakly Krull domain $D$ is an AWFD if and only if $\mathrm{Cl}(D)$ is torsion. We note that $t-\operatorname{dim}(D[\Gamma])=t-\operatorname{dim}(D[X])$ for any numerical semigroup $\Gamma$ [Chang et al. 2012, Theorem 1.5].

Let $\mathbb{N}_{0}$ (resp., $\mathbb{Z}$ ) be the set of nonnegative integers (resp., integers). A semigroup $\Gamma$ is called a numerical semigroup if $\Gamma$ is a subset of $\mathbb{N}_{0}$ containing 0 and generates $\mathbb{Z}$ as a group. It is known that if $\Gamma$ is a numerical semigroup, then $\Gamma$ is finitely generated and $\mathbb{N}_{0} \backslash \Gamma$ is a finite set. Hence there exists the largest nonnegative integer which is not contained in $\Gamma$. This number is called the Frobenius number of $\Gamma$ and is denoted by $F(\Gamma)$.

Throughout this article, $D \subseteq E$ denotes an extension of integral domains, $q f(D)$ (resp., $q f(E)$ ) is the quotient field of $D$ (resp., $E$ ), $\bar{D}$ means the integral closure of $D, X$ is an indeterminate over $E, \Gamma$ is a numerical semigroup with $\Gamma \subsetneq \mathbb{N}_{0}$ and $D[\Gamma]$ is the numerical semigroup ring of $\Gamma$ over $D$. Note that each element $f \in D[\Gamma]$ is uniquely expressible in the form $f=a_{1} X^{\alpha_{1}}+\cdots+a_{k} X^{\alpha_{k}}$, where $a_{i} \in D$ and $\alpha_{i} \in \Gamma$ with $\alpha_{1}<\cdots<\alpha_{k}$. Let $\Gamma^{*}=\Gamma \backslash\{0\}, R=D+E\left[\Gamma^{*}\right], T=D+X E[X]$ and $T_{n}=D+X^{n} E[X]$ for integers $n \geq 2$, i.e., $R=\{f \in E[\Gamma] \mid f(0) \in D\}$, $T=\{f \in E[X] \mid f(0) \in D\}$ and $T_{n}=R$ when $\Gamma=\{0\} \cup\left\{m \in \mathbb{N}_{0} \mid m \geq n\right\}$. Then $D[\Gamma] \subseteq R \subseteq E[\Gamma]$ and $T_{F(\Gamma)+1} \subseteq R \subsetneq T \subseteq E[X]$. For an $f \in q f(D)[\Gamma], c(f)$ means the fractional ideal of $D$ generated by the coefficients of $f$. If $I$ is an ideal of $D[\Gamma]$, then $c(I)$ denotes the ideal of $D$ generated by the coefficients of all the polynomials in $I$.

In multiplicative ideal theory, the $D+E\left[\Gamma^{*}\right]$ construction has been extensively studied by several authors for its interest in constructing examples with prescribed properties. As a special kind of pullbacks, this has become so important that in recent years there have been many papers devoted to ring- and ideal-theoretic properties in this construction.

Anderson et al. [2003a; 2006] (see also [Anderson and Chang 2007]) studied when the domains $D\left[X^{2}, X^{3}\right], D+X E[X]$ and $D+X^{2} E[X]$ are weakly Krull 
domains, WFDs, AWFDs or GWFDs. In fact, they showed that $D\left[X^{2}, X^{3}\right]$ is a weakly Krull domain if and only if $D$ is a weakly Krull UMT-domain [Anderson et al. 2003a, Proposition 2.7]; if $\operatorname{char}(D) \neq 0$, then $D\left[X^{2}, X^{3}\right]$ is an AWFD if and only if $D\left[X^{2}, X^{3}\right]$ is a GWFD [Anderson and Chang 2007, Corollary 2.11]; $D+X E[X]$ is a weakly Krull domain if and only if $D+X^{2} E[X]$ is a weakly Krull domain [Anderson et al. 2006, Theorem 4.3]; and $D+X E[X]$ is an AWFD if and only if $D+X E[X]$ is a GWFD [Anderson and Chang 2007, Corollary 2.10]. The main purpose of this paper is to determine how certain properties of $D, E$ and $\Gamma$ influence those of $R$, and vice versa. This also extends the results for the domains $D\left[X^{2}, X^{3}\right], D+X E[X]$ and $D+X^{2} E[X]$ to any composite numerical semigroup ring $D+E\left[\Gamma^{*}\right]$.

In Section 1, we investigate weakly Krull domains, AWFDs and GWFDs in the context of numerical semigroup rings $D[\Gamma]$ which coincide with the domains $R=D+E\left[\Gamma^{*}\right]$ when $D=E$. We prove that $D[\Gamma]$ is a weakly Krull domain if and only if $D$ is a weakly Krull UMT-domain, and that if $\operatorname{char}(D) \neq 0$, then $D[\Gamma]$ is an AWFD if and only if $D[\Gamma]$ is a GWFD, if and only if $D$ is an almost weakly factorial quasi-AGCD-domain, if and only if $D$ is a generalized weakly factorial quasi-AGCD-domain.

In Section 2, we study when the domain $R=D+E\left[\Gamma^{*}\right]$ is a weakly Krull domain, an AWFD or a GWFD, where $D \subsetneq E$. We show that $R$ is a weakly Krull domain if and only if $T=D+X E[X]$ is a weakly Krull domain, and that if $\operatorname{char}(E) \neq 0$, then $R$ is an AWFD if and only if $R$ is a GWFD, if and only if $T$ is an AWFD, if and only if $R$ is a GWFD. We also prove that $R$ is never a WFD.

\section{Weakly Krull domains as numerical semigroup rings}

In this section, we characterize when the numerical semigroup ring $D[\Gamma]$ is a weakly Krull domain, an AWFD or a GWFD.

The first two lemmas are well known for the general semigroup rings, but we include their proofs for the convenience of the reader.

Lemma 1.1 [El Baghdadi et al. 2002, Lemma 2.3]. Let D be an integral domain and $\Gamma$ be a numerical semigroup. The following statements hold for an $I \in \mathbf{F}(D)$ :

(1) $(I D[\Gamma])^{-1}=I^{-1} D[\Gamma]$.

(2) $(I D[\Gamma])_{v}=I_{v} D[\Gamma]$.

(3) $(I D[\Gamma])_{t}=I_{t} D[\Gamma]$.

Proof. (1) Since $(I D[\Gamma])\left(I^{-1} D[\Gamma]\right) \subseteq D[\Gamma], I^{-1} D[\Gamma] \subseteq(I D[\Gamma])^{-1}$. Conversely, let $f \in(I D[\Gamma])^{-1}$. Then $f I D[\Gamma] \subseteq D[\Gamma]$ and hence $c(f) I \subseteq D$. Hence $c(f) \subseteq$ $I^{-1}$, and therefore $f \in c(f) D[\Gamma] \subseteq I^{-1} D[\Gamma]$. Thus the equality holds.

(2) By (1), $(I D[\Gamma])_{v}=\left((I D[\Gamma])^{-1}\right)^{-1}=\left(I^{-1} D[\Gamma]\right)^{-1}=I_{v} D[\Gamma]$. 
(3) Let $f_{1}, \ldots, f_{n}$ be nonzero elements of $I D[\Gamma]$. Then we have

$$
\begin{aligned}
\left(\left(f_{1}, \ldots, f_{n}\right) D[\Gamma]\right)_{v} & \subseteq\left(\left(c\left(f_{1}\right), \ldots, c\left(f_{n}\right)\right) D[\Gamma]\right)_{v} \\
& =\left(c\left(f_{1}\right), \ldots, c\left(f_{n}\right)\right)_{v} D[\Gamma] \\
& \subseteq I_{t} D[\Gamma]
\end{aligned}
$$

by (2), i.e., $(I D[\Gamma])_{t} \subseteq I_{t} D[\Gamma]$. For the reverse inclusion, let $J$ be a nonzero finitely generated subideal of $I$. Then $J_{v} D[\Gamma]=(J D[\Gamma])_{v} \subseteq(I D[\Gamma])_{t}$ by (2). Hence $I_{t} D[\Gamma] \subseteq(I D[\Gamma])_{t}$. Thus we have the desired equality.

Lemma 1.2 [Anderson and Chang 2005, Corollary 2.3]. Let D be an integral domain, $\Gamma$ be a numerical semigroup and let $Q$ be a maximal $t$-ideal of $D[\Gamma]$ such that $Q \cap D \neq(0)$. Then $Q=(Q \cap D) D[\Gamma]$. In particular, $Q \cap D$ is a maximal $t$-ideal of $D$.

Proof. The containment $(Q \cap D) D[\Gamma] \subseteq Q$ is obvious. For the converse, it suffices to show that $c(Q) \subseteq Q$. Suppose to the contrary that $c(Q) \nsubseteq Q$. Then

$$
Q \subsetneq c(Q) D[\Gamma] .
$$

Since $Q$ is a maximal $t$-ideal of $D[\Gamma],(c(Q) D[\Gamma])_{t}=D[\Gamma]$. Therefore $c(Q)_{t}=D$ by Lemma 1.1(3), and hence $c(f)_{v}=D$ for some $f \in Q$. Let $0 \neq d \in Q \cap D$ and choose $0 \neq g \in(d, f)^{-1}$. Then $g d \in D[\Gamma]$ and hence $g \in q f(D)[\Gamma]$. Also, we have $f g \in D[\Gamma]$. Hence it follows from [Gilmer 1992, Theorem 28.1] that

$$
c(g) \subseteq c(g)_{v}=\left(c(f)^{m+1} c(g)\right)_{v}=\left(c\left(f^{m}\right) c(f g)\right)_{v}=c(f g)_{v} \subseteq D,
$$

where $m$ is the degree of $g$. So $g \in c(g) D[\Gamma] \subseteq D[\Gamma]$, which implies that $(d, f)^{-1}=$ $D[\Gamma]$. This contradicts the fact that $Q$ is a maximal $t$-ideal of $D[\Gamma]$. Therefore $c(Q) \subseteq Q$, and thus $Q \subseteq(Q \cap D) D[\Gamma]$. The second assertion is an immediate consequence of Lemma 1.1(3).

An integral domain $B$ is said to be a UMT-domain if every upper to zero (a nonzero prime ideal of $B[X]$ which contracts to zero in $B) Q$ of $B[X]$ is a maximal $t$-ideal (equivalently, is $t$-invertible). Now, we give the numerical semigroup ring version of [Anderson et al. 1993, Proposition 4.11].

Theorem 1.3. Let $D$ be an integral domain and $\Gamma$ be a numerical semigroup with $\Gamma \subsetneq \mathbb{N}_{0}$. Then the following assertions are equivalent.

(1) $D[\Gamma]$ is a weakly Krull domain.

(2) $D[X]$ is a weakly Krull domain.

(3) D is a weakly Krull UMT-domain. 
Proof. (1) $\Rightarrow$ (3) Assume $D[\Gamma]$ is a weakly Krull domain. Then $t-\operatorname{dim}(D[\Gamma])=1$ [Anderson et al. 1992, Lemma 2.1]. Let $P$ be a prime $t$-ideal of $D$. Then $P D[\Gamma]$ is a prime $t$-ideal of $D[\Gamma]$ by Lemma 1.1(3); so ht ${ }_{D}(P)=\mathrm{ht}_{D[\Gamma]}(P D[\Gamma])=1$; so $t-\operatorname{dim}(D)=1$. Since $t-\operatorname{dim}(D[\Gamma])=1$, we have $t-\operatorname{dim}(D[X])=1$ by [Chang et al. 2012, Theorem 1.5]. Therefore every upper to zero in $D[X]$ is a maximal $t$-ideal, and thus $D$ is a UMT-domain. Note that

$$
D=\bigcap_{P \in X^{1}(D)} D_{P}
$$

by [Kang 1989, Proposition 2.9]. To show that this intersection has finite character, let $d \in D \backslash\{0\}$. Since $D[\Gamma]$ is a weakly Krull domain, $d$ belongs to only finitely many height-one prime ideals of $D[\Gamma]$, and hence there exists only a finite number of height-one prime ideals of $D$ containing $d$. Thus $D$ is a weakly Krull domain.

(3) $\Rightarrow$ (1) Assume that $D$ is a weakly Krull UMT-domain and let $Q$ be a maximal $t$-ideal of $D[\Gamma]$ with $Q \cap D \neq(0)$. By Lemma 1.2, $Q=(Q \cap D) D[\Gamma]$ and $Q \cap D$ is a maximal $t$-ideal of $D$. Since $t-\operatorname{dim}(D)=1$ [Anderson et al. 1992, Lemma 2.1], $\mathrm{ht}_{D}(Q \cap D)=1$; so ht $D[\Gamma] Q \leq 2$ (cf. [Kaplansky 1970, Theorem 37]). If ht $_{D[\Gamma]} Q=2$, then there exists a nonzero prime ideal $P \subsetneq Q$ which contracts to zero in $D$. Note that $P=M \cap D[\Gamma]$ for some prime ideal $M$ of $D[X]$ [Chang et al. 2012, Proposition 1.1]. Since $M \cap D=(0)$ and $D$ is a UMT-domain, $M$ is a maximal $t$-ideal of $D[X]$. Hence $P$ is a maximal $t$-ideal of $D[\Gamma]$ [Chang et al. 2012, Theorem 1.4]. This contradicts the choice of $P$. Thus $t-\operatorname{dim}(D[\Gamma])=1$. By [Kang 1989, Proposition 2.9], we have $D[\Gamma]=\bigcap_{Q \in X^{1}(D[\Gamma])} D[\Gamma]_{Q}$. We claim that this intersection has finite character. Let $f \in D[\Gamma] \backslash\{0\}$ and set

$$
\begin{aligned}
\mathscr{S} & =\left\{Q \in X^{1}(D[\Gamma]) \mid f \in Q\right\}, \\
\mathscr{S}_{1} & =\left\{Q \in \mathscr{Y} \mid Q \cap D \in X^{1}(D)\right\}, \text { and } \\
\mathscr{S}_{2} & =\{Q \in \mathscr{Y} \mid Q \cap D=(0)\} .
\end{aligned}
$$

Then $\mathscr{S}=\mathscr{Y}_{1} \cup \mathscr{Y}_{2}$. If $\mathscr{Y}_{1}$ is an infinite set, then $c(f)$ belongs to infinitely many height-one prime ideals of $D$ by Lemma 1.2. This is absurd, because $D$ is a weakly Krull domain. Hence $\mathscr{Y}_{1}$ is a finite set. Note that $q f(D)[\Gamma]$ is a one-dimensional Noetherian domain; so $q f(D)[\Gamma]$ is a weakly Krull domain. Hence $\mathscr{Y}_{2}$ is also a finite set. Therefore $\mathscr{Y}$ is a finite set. Thus $D[\Gamma]$ is a weakly Krull domain.

(2) $\Leftrightarrow$ (3) See [Anderson et al. 1993, Proposition 4.11].

Recall that if $D \subseteq E$ is an extension of integral domains, then $E$ is said to be a root extension of $D$ if for each $z \in E$, there is a positive integer $n=n(z)$ such that $z^{n} \in D$. A domain $B$ is called an almost Prüfer $v$-multiplication domain (APv $\mathrm{MD}$ ) (resp., almost GCD-domain (AGCD-domain)) if for each $0 \neq a, b \in B$, there exists a positive integer $n=n(a, b)$ such that $\left(a^{n}, b^{n}\right)_{v}$ is $t$-invertible (resp., principal). 
It is known that $B$ is a weakly Krull $\mathrm{P} v \mathrm{MD}$ if and only if $B[X]$ is weakly Krull and $B$ is integrally closed [Anderson et al. 1993, Corollary 4.13]. We weaken the hypothesis and obtain the following result.

Corollary 1.4. Let $D$ be an integral domain and $\Gamma$ be a numerical semigroup.

(1) D is a weakly Krull APvMD if and only if $D[\Gamma]$ is a weakly Krull domain and $D \subseteq \bar{D}$ is a root extension.

(2) $D$ is an almost weakly factorial $A G C D$-domain if and only if $D[\Gamma]$ is a weakly Krull domain, $\mathrm{Cl}(D)$ is torsion and $D \subseteq \bar{D}$ is a root extension.

Proof. (1) By [Li 2012, Theorem 3.8], a domain $B$ is an APvMD if and only if $B$ is a UMT-domain and $B \subseteq \bar{B}$ is a root extension. Thus the result follows from Theorem 1.3.

(2) By [Li 2012, Theorem 3.1], a domain $B$ is an AGCD-domain if and only if $B$ is an $\mathrm{AP} v \mathrm{MD}$ and $\mathrm{Cl}(B)$ is torsion. Also, by [Anderson et al. 1992, Theorem 3.4], $B$ is an AWFD if and only if $B$ is a weakly Krull domain and $\mathrm{Cl}(B)$ is torsion. Thus the result is an immediate consequence of Theorem 1.3 and (1).

Let $S$ be a saturated multiplicative subset of a domain $B$ and let $N(S)=\{0 \neq b \in$ $B \mid(b, s)_{v}=B$ for all $\left.s \in S\right\}$ be the $m$-complement of $S$. We say that $S$ is an almost splitting set if for each $0 \neq b \in B$, there exists a positive integer $n=n(b)$ such that $b^{n}=s t$ for some $s \in S$ and $t \in N(S)$. Following [Anderson and Chang 2007], $B$ is called a quasi-AGCD-domain if $B \backslash\{0\}$ is an almost splitting set in $B[X]$. It was shown that if $B$ is integrally closed, then the notion of quasi-AGCD-domains coincides with that of AGCD-domains [Chang 2005, Proposition 2.6]. The next corollary characterizes when the numerical semigroup ring $D[\Gamma]$ is an AWFD or a GWFD.

Corollary 1.5. Let $D$ be an integral domain with $\operatorname{char}(D) \neq 0$ and $\Gamma$ be a numerical semigroup with $\Gamma \subsetneq \mathbb{N}_{0}$. Then the following conditions are equivalent.

(1) $D[\Gamma]$ is an $A W F D$.

(2) $D[\Gamma]$ is a GWFD.

(3) $D[X]$ is an AWFD.

(4) $D[X]$ is a GWFD.

(5) $D$ is an almost weakly factorial quasi-AGCD-domain.

(6) $D$ is a generalized weakly factorial quasi-AGCD-domain.

(7) D is a weakly Krull quasi-AGCD-domain.

Proof. Let $\operatorname{char}(D)=p$.

$(1) \Rightarrow(2)$ This is well known. 
(1) $\Leftrightarrow$ (3) By [Anderson et al. 1992, Theorem 3.4], an integral domain $B$ is an AWFD if and only if $B$ is a weakly Krull domain and $\mathrm{Cl}(B)$ is torsion, and by Theorem $1.3, D[\Gamma]$ is a weakly Krull domain if and only if $D[X]$ is a weakly Krull domain. By [Chang et al. 2012, Lemma 2.7], Pic $(q f(D)[\Gamma])$ is torsion if and only if $\operatorname{char}(D) \neq 0$. Since $\mathrm{Cl}(D[\Gamma])=\mathrm{Cl}(D[X]) \oplus \operatorname{Pic}(q f(D)[\Gamma])$ [Anderson and Chang 2004, Theorem 5], $\mathrm{Cl}(D[\Gamma])$ is torsion if and only if $\mathrm{Cl}(D[X])$ is torsion and $\operatorname{char}(D) \neq 0$. Thus this equivalence follows from these facts.

$(4) \Rightarrow$ (2) By [Anderson et al. 2003b, Theorem 2.2], a domain $B$ is a GWFD if and only if $t-\operatorname{dim}(B)=1$ and for each $P \in X^{1}(B), P=\sqrt{b B}$ for some $b \in B$. Assume that $D[X]$ is a GWFD and let $P \in X^{1}(D[\Gamma])$. Since $t-\operatorname{dim}(D[\Gamma])=t-\operatorname{dim}(D[X])=$ 1 [Chang et al. 2012, Theorem 1.5], it suffices to show that $P=\sqrt{f D[\Gamma]}$ for some $f \in D[\Gamma]$. If $P \cap D \neq(0)$, then $P=(P \cap D) D[\Gamma]$ by Lemma 1.2. Since $D[X]$ is a GWFD, $(P \cap D) D[X]=\sqrt{d D[X]}$ for some $d \in P \cap D$. It is easy to see that $P=\sqrt{d D[\Gamma]}$. Next, suppose that $P \cap D=(0)$. Then there exists a prime $t$-ideal $Q$ of $D[X]$ such that $P=Q \cap D[\Gamma]$ [Chang et al. 2012, Theorem 1.5]. Since $D[X]$ is a GWFD, $Q=\sqrt{f D[X]}$ for some $f \in D[X]$. Also, since $\operatorname{char}(D)=p>0$, there exists a positive integer $n$ such that $f^{p^{n}} \in D[\Gamma]$. An easy calculation shows that $P=\sqrt{f p^{n} D[\Gamma]}$. Thus $D[\Gamma]$ is a GWFD.

$(2) \Rightarrow(4)$ This direction is an easy modification of the proof of (4) $\Rightarrow$ (2).

(2) $\Rightarrow(5)$ See [Anderson and Chang 2007, Corollary 2.9].

$(5) \Rightarrow(6) \Rightarrow(7)$ These implications are obvious.

(7) $\Rightarrow$ (1) Assume that $D$ is a weakly Krull quasi-AGCD-domain. Then $D$ is a UMT-domain and $\mathrm{Cl}(D[X])$ is torsion [Anderson and Chang 2007, Theorem 2.4]. Hence $D[\Gamma]$ is a weakly Krull domain by Theorem 1.3. Also, it follows from [Anderson and Chang 2004, Theorem 5; Chang et al. 2012, Lemma 2.7] that $\mathrm{Cl}(D[\Gamma])$ is torsion. Thus $D[\Gamma]$ is an AWFD [Anderson et al. 1992, Theorem 3.4].

We end this section by noting that $D[\Gamma]$ is never a WFD. We also show that $D[\Gamma]$ need not be an AWFD if $\operatorname{char}(D)=0$.

Remark 1.6. (1) Let $B$ be an integral domain with quotient field $K$. In [Gilmer and Martin 1990, Theorem 7], Gilmer and Martin showed that if $B$ is a seminormal domain and $B+X^{n} B[X] \subseteq B[\Gamma]$, then $\operatorname{Pic}(B[\Gamma])=\operatorname{Pic}(B) \oplus\left(W_{n} / L\right)$, where $L \subseteq$ $W_{n}$ are the subgroups of the group $U\left(B[X] / X^{n} B[X]\right)$ of units of $B[X] / X^{n} B[X]$ defined by $W_{n}=\left\{1+X f+X^{n} B[X] \mid f \in B[X]\right\}$ and $L=\left\{1+X f+X^{n} B[X] \mid\right.$ $1+X f \in B[\Gamma]\}$. Note that $\mathrm{Cl}(B[\Gamma])=\mathrm{Cl}(B[X]) \oplus \operatorname{Pic}(K[\Gamma])$ [Anderson and Chang 2004, Theorem 5] and that $B$ is a WFD if and only if $B$ is a weakly Krull domain and $\mathrm{Cl}(B)=0$ [Anderson and Zafrullah 1990, Theorem]. If $D[\Gamma]$ is a WFD, then $\operatorname{Cl}(D[\Gamma])=0$, and hence $\operatorname{Pic}(q f(D)[\Gamma])=0$. Therefore $W_{n}=L$; 
so $1+X+X^{n} q f(D)[X] \in L$, which implies that $1 \in \Gamma$. Thus, if $\Gamma$ is a proper numerical semigroup, then $D[\Gamma]$ is never a WFD.

(2) If $D[\Gamma]$ is an AWFD, then $\mathrm{Cl}(D[\Gamma])$ is torsion [Anderson et al. 1992, Theorem 3.4]; so Pic $(q f(D)[\Gamma])$ is torsion [Anderson and Chang 2004, Theorem 5]. Hence $\operatorname{char}(D) \neq 0$ [Chang et al. 2012, Lemma 2.7]. This shows that the condition that $\operatorname{char}(D) \neq 0$ is essential in Corollary 1.5.

(3) It is known that a generalized unique factorization domain (GUFD) is a weakly factorial GCD-domain [Anderson et al. 1995, Theorem 7], and hence integrally closed. (See [Anderson et al. 1995] for the definition and some characterizations of a GUFD.) Thus, if $\Gamma$ is a numerical semigroup with $\Gamma \subsetneq \mathbb{N}_{0}$, then $D[\Gamma]$ is not a GUFD by (1). In fact, $D[\Gamma]$ is not integrally closed; so $D[\Gamma]$ is never a GUFD.

\section{Weakly Krull domains and the ring $D+E\left[\Gamma^{*}\right]$ when $D \subset E$}

For a domain $A, \operatorname{Spec}(A)$ stands for the set of prime ideals of $A$. Assume that $D \subsetneq E$ is an extension of integral domains, $\Gamma$ is a numerical semigroup with $\Gamma \subsetneq \mathbb{N}_{0}$ and let $R=D+E\left[\Gamma^{*}\right], T=D+X E[X], T_{n}=D+X^{n} E[X]$ and $\Delta_{n}=\{0\} \cup$ $\left\{m \in \mathbb{N}_{0} \mid m \geq n\right\}$ for integers $n \geq 2$. Note that $D[\Gamma] \subsetneq R \subsetneq T$ and $T_{n} \subsetneq T$. In this section, we characterize when the domains $R$ and $T_{n}$ are weakly Krull domains, AWFDs or GWFDs. To do this, we need two lemmas.

Lemma 2.1. Let $R=D+E\left[\Gamma^{*}\right]$ and $T=D+X E[X]$. If $Q$ is a prime ideal of $R$, then there exists a unique prime ideal of $T$ lying over $Q$. Thus the natural map $\phi: \operatorname{Spec}(T) \rightarrow \operatorname{Spec}(R)$, given by $P \mapsto P \cap R$, is an order-preserving bijection. In particular, $h t_{T}(X E[X])=h t_{R}\left(E\left[\Gamma^{*}\right]\right)$.

Proof. Let $Q$ be a prime ideal of $R$. Since $T$ is an integral extension of $R$, there exists a prime ideal $P$ of $T$ such that $Q=P \cap R$ [Kaplansky 1970, Theorem 44]. Note that $E\left[\Gamma^{*}\right] \subseteq Q$ if and only if $X E[X] \subseteq P$. If $E\left[\Gamma^{*}\right] \subseteq Q$, then $P$ is the unique prime ideal of $T$ lying over $Q$ because $R / X E[X] \cong D \cong R / E\left[\Gamma^{*}\right]$. If $E\left[\Gamma^{*}\right] \nsubseteq Q Q$, then $X^{F(\Gamma)+1} f \notin Q$ for some $f \in E[X]$; so

$$
g=\frac{X^{F(\Gamma)+1} f g}{X^{F(\Gamma)+1} f} \in R_{Q}
$$

for any $g \in T$. Hence $T_{Q R_{Q} \cap T}=R_{Q}$. Thus $Q R_{Q} \cap T$ is the unique prime ideal of $T$ lying over $Q$.

Let $n$ be an integer $\geq 2$. Then it is clear that if $\Gamma=\Delta_{n}$, then $R=T_{n}$. Hence Lemma 2.1 also shows that $\mathrm{ht}_{T}(X E[X])=\mathrm{ht}_{T_{n}}\left(X^{n} E[X]\right)$.

Remark 2.2. Let $\Gamma=\left\{\alpha_{1}, \ldots, \alpha_{n}\right\} \cup \Delta_{F(\Gamma)+1}$ with $1<\alpha_{1}<\cdots<\alpha_{n}<F(\Gamma)+1$ and $R=D+E\left[\Gamma^{*}\right]$. 
(1) Let $g \in\left(R: E\left[\Gamma^{*}\right]\right)$. Then $g E\left[\Gamma^{*}\right] \subseteq R$; hence for each $\alpha \in \Gamma^{*}, g X^{\alpha}=a_{\alpha}+f_{\alpha}$ for some $a_{\alpha} \in D$ and $f_{\alpha} \in E\left[\Gamma^{*}\right]$. Therefore $g X^{\alpha+F(\Gamma)}=\left(a_{\alpha}+f_{\alpha}\right) X^{F(\Gamma)} \in R$, which means that $a_{\alpha}=0$. Hence $g X^{\alpha}=f_{\alpha} \in E\left[\Gamma^{*}\right]$, and so $g \in \bigcap_{\alpha \in \Gamma^{*}}\left\{\frac{1}{X^{\alpha}} f \mid f \in E\left[\Gamma^{*}\right]\right\}$. The reverse containment is obvious. Thus we have

$$
\left(R: E\left[\Gamma^{*}\right]\right)=\bigcap_{\alpha \in \Gamma^{*}}\left\{\frac{1}{X^{\alpha}} f \mid f \in E\left[\Gamma^{*}\right]\right\} .
$$

(2) It is clear that $E[\Gamma] \subsetneq\left(R: E\left[\Gamma^{*}\right]\right)$ because $X^{F(\Gamma)} \in\left(R: E\left[\Gamma^{*}\right]\right) \backslash E[\Gamma]$. Let $g \in\left(R: E\left[\Gamma^{*}\right]\right)$. Then $X^{F(\Gamma)+1} g \in R$; so we can write

$$
X^{F(\Gamma)+1} g=\sum_{i=0}^{n} g_{i} X^{\alpha_{i}}+X^{F(\Gamma)+1} h
$$

for some $g_{i} \in E$ and $h \in E[X]$. (For the sake of convenience, set $\alpha_{0}=0$.). Fix a $k \in\{1, \ldots, n\}$. Then we have $X^{2 F(\Gamma)-\alpha_{k}+1} g=\sum_{i=0}^{k-1} g_{i} X^{F(\Gamma)+\alpha_{i}-\alpha_{k}}+g_{k} X^{F(\Gamma)}+$ $X^{F(\Gamma)+1}\left(\sum_{i=k+1}^{n} g_{i} X^{\alpha_{i}-\alpha_{k}-1}+h\right) \in R$; so $g_{k}=0$ for all $k=1, \ldots, n$. Also, we have $X^{F(\Gamma)+2} g=g_{0} X+X^{F(\Gamma)+2} h \in R$; so $g_{0}=0$. Therefore $X^{F(\Gamma)+1} g=X^{F(\Gamma)+1} h$, and hence $g=h \in E[X]$. Thus $E[\Gamma] \subsetneq\left(R: E\left[\Gamma^{*}\right]\right) \subseteq E[X]$. In particular, if $\Gamma=\Delta_{F(\Gamma)+1}$, then $E[X] \subseteq\left(R: E\left[\Gamma^{*}\right]\right) ;$ so $\left(R: E\left[\Gamma^{*}\right]\right)=E[X]$.

(3) Lemma 4.2 of [Anderson et al. 2006] cannot be extended to any proper numerical semigroup, i.e., it may happen that $\left(R: E\left[\Gamma^{*}\right]\right) \subsetneq E[X]$ for some $\Gamma \subsetneq \mathbb{N}_{0}$. For instance, if $\Gamma=\{2\} \cup \Delta_{4}$, then $X \in E[X] \backslash\left(R: E\left[\Gamma^{*}\right]\right)$.

Lemma 2.3. The following statements hold for $R=D+E\left[\Gamma^{*}\right]$.

(1) $E\left[\Gamma^{*}\right]$ is a prime $t$-ideal of $R$.

(2) $E\left[\Gamma^{*}\right]$ is a maximal $t$-ideal of $R$ if and only if $q f(D) \cap E=D$.

Proof. (1) Let $\Gamma=\left\{\alpha_{1}, \ldots, \alpha_{k}\right\} \cup \Delta_{F(\Gamma)+1}$ such that $0<\alpha_{1}<\cdots<\alpha_{k}<F(\Gamma)+1$. Since $R / E\left[\Gamma^{*}\right] \cong D, E\left[\Gamma^{*}\right]$ is a prime ideal of $R$. It suffices to show that $E\left[\Gamma^{*}\right]$ is a $v$-ideal of $R$, because each $v$-ideal is a $t$-ideal.

Case 1. $\left\{\alpha_{1}, \ldots, \alpha_{k}\right\}$ is empty. In this case, $\left(R: E\left[\Gamma^{*}\right]\right)=E[X]$ by Remark 2.2(2); so we need to show that $(R: E[X])=E\left[\Gamma^{*}\right]$. It is clear that $E\left[\Gamma^{*}\right] \subseteq(R: E[X])$. For the converse, let $f \in(R: E[X])$. Then $f E[X] \subseteq R$. Since $1 \in E[X], f \in R$. Also, since $X \in E[X], f(0)=0$; so $f \in E\left[\Gamma^{*}\right]$.

Case 2. $\left\{\alpha_{1}, \ldots, \alpha_{k}\right\}$ is nonempty. Deny the conclusion, and then there exists a polynomial $g=g_{0}+\sum_{i=1}^{k} g_{\alpha_{i}} X^{\alpha_{i}}+\sum_{i=F(\Gamma)+1}^{l} g_{i} X^{i} \in\left(E\left[\Gamma^{*}\right]\right)_{v} \backslash E\left[\Gamma^{*}\right]$. Hence $g\left(R: E\left[\Gamma^{*}\right]\right) \subseteq R$. Let $f \in\left(R: E\left[\Gamma^{*}\right]\right)$. Then $f \in E[X]$ by Remark 2.2(2); so we can write $f=\sum_{i=0}^{m} f_{i} X^{i}$. Note that

$$
f g=f_{0} g_{0}+g_{0} \sum_{i=1}^{\alpha_{1}-1} f_{i} X^{i}+\left(f_{0} g_{\alpha_{1}}+f_{\alpha_{1}} g_{0}\right) X^{\alpha_{1}}+X^{\alpha_{1}+1} h_{1}
$$


for some $h_{1} \in E[X]$. Since $f g \in R$ and $g_{0} \neq 0, f_{1}=\cdots=f_{\alpha_{1}-1}=0$; so $f=$ $f_{0}+\sum_{i=\alpha_{1}}^{m} f_{i} X^{i}$. Note that $2 \alpha_{1} \in \Gamma^{*}$; so $2 \alpha_{1} \geq F(\Gamma)+1$ or $2 \alpha_{1}=\alpha_{p}$ for some $p \in\{2, \ldots, k\}$. If $2 \alpha_{1} \geq F(\Gamma)+1$, then we have

$f g=f_{0} g_{0}+\left(f_{0} g_{\alpha_{1}}+f_{\alpha_{1}} g_{0}\right) X^{\alpha_{1}}+g_{0} \sum_{i=\alpha_{1}+1}^{\alpha_{2}-1} f_{i} X^{i}+\left(f_{0} g_{\alpha_{2}}+f_{\alpha_{2}} g_{0}\right) X^{\alpha_{2}}+X^{\alpha_{2}+1} h_{2}$

for some $h_{2} \in E[X]$. Again, since $f g \in R, f_{\alpha_{1}+1}=\cdots=f_{\alpha_{2}-1}=0$. By repeating this process, we have $f_{i}=0$ for all $i \in \mathbb{N}_{0} \backslash \Gamma$, and hence $f \in R$. Therefore $\left(R: E\left[\Gamma^{*}\right]\right)=R$. However, this is impossible because $X^{F(\Gamma)} \in\left(R: E\left[\Gamma^{*}\right]\right) \backslash R$. If $2 \alpha_{1}=\alpha_{p}$ for some $p \in\{2, \ldots, k\}$, a simple modification of the proof of the previous case leads to the same conclusion because $2 \alpha_{l} \geq F(\Gamma)+1$ for some $l \leq k$. In either case, $E\left[\Gamma^{*}\right]$ is a $v$-ideal, and thus $E\left[\Gamma^{*}\right]$ is a $t$-ideal of $R$.

(2) This appears in [Lim 2012, Lemma 1.2].

Now, we are ready to give a necessary and sufficient condition for the domain $R$ to be a weakly Krull domain.

Theorem 2.4. Let $R=D+E\left[\Gamma^{*}\right], T=D+X E[X], T_{n}=D+X^{n} E[X]$ and $\Delta_{n}=\{0\} \cup\left\{m \in \mathbb{N}_{0} \mid m \geq n\right\}$ for integers $n \geq 2$. Then the following statements are equivalent.

(1) $R$ is a weakly Krull domain.

(2) $T$ is a weakly Krull domain.

(3) $T_{n}$ is a weakly Krull domain.

(4) $X^{n} E[X]$ is a height-one maximal t-ideal of $T_{n}$ and $E\left[\Delta_{n}\right]$ is a weakly Krull domain.

(5) $E_{D \backslash\{0\}}$ is a field, $q f(D) \cap E=D$ and $E[X]$ is a weakly Krull domain.

Proof. (2) $\Rightarrow$ (1) Let $T$ be a weakly Krull domain. Let $\Gamma=\left\{\alpha_{1}, \ldots, \alpha_{k}\right\} \cup \Delta_{F(\Gamma)+1}$ be such that $0<\alpha_{1}<\cdots<\alpha_{k}<F(\Gamma)+1$. Then $T=\bigcap_{P \in X^{1}(T)} T_{P}$ and this intersection has finite character. Note that $X E[X]$ is a height-one prime ideal of $T$ [Anderson et al. 2006, Theorem 3.4]; so $E\left[\Gamma^{*}\right]$ is a height-one prime ideal of $R$ by Lemma 2.1. We claim that $R=\bigcap_{P \cap R \in X^{1}(R)} R_{P \cap R}$, where $P$ ranges over all heightone prime ideals of $T$. Suppose to the contrary that there exists an element $f$ in $\bigcap_{P \cap R \in X^{1}(R)} R_{P \cap R} \backslash R$. Note that $f \in T$, and hence we can write $f=\sum_{i=0}^{m} f_{i} X^{i}$. Then there exists a polynomial $g \in R \backslash E\left[\Gamma^{*}\right]$ such that $f g \in R$. Since $g(0) \neq 0$, the same argument as in the proof of Lemma 2.3(1) shows that $f \in R$, which contradicts the choice of $f$. Thus the equality holds. Since $T=\bigcap_{P \in X^{1}(T)} T_{P}$ has finite character, it is clear that the intersection $R=\bigcap_{P \cap R \in X^{1}(R)} R_{P \cap R}$ also has finite character. Thus $R$ is a weakly Krull domain.

$(2) \Rightarrow$ (3) This implication was already shown in the proof of (2) $\Rightarrow$ (1). 
(3) $\Rightarrow$ (4) Assume that $T_{n}$ is a weakly Krull domain. Then $t-\operatorname{dim}\left(T_{n}\right)=1$ [Anderson et al. 1992, Lemma 2.1]; so $X^{n} E[X]$ is a maximal $t$-ideal of $T_{n}$ by Lemma 2.3(1).

Let $S=\left\{X^{m} \mid m \in \Delta_{n}\right\}$. Then $E\left[\Delta_{n}\right]_{S}=E\left[X, X^{-1}\right]=\left(T_{n}\right)_{S}$ is a weakly Krull domain [Anderson et al. 1993, Proposition 4.7]. Note that $X E[X]$ is a height-one prime ideal of $E[X]$; so $X^{n} E[X]$ is a height-one prime ideal of $E\left[\Delta_{n}\right]$ [Chang et al. 2012, Proposition 1.1]; so $E\left[\Delta_{n}\right]_{X^{n} E[X]}$ is a one-dimensional quasi-local domain. Hence $E\left[\Delta_{n}\right]_{X^{n} E[X]}$ is a weakly Krull domain. We claim that $E\left[\Delta_{n}\right]=$ $E\left[\Delta_{n}\right]_{S} \cap E\left[\Delta_{n}\right]_{X^{n} E[X]}$. Let $f=f_{0}+\sum_{i=n}^{k_{1}} f_{i} X^{i}$ and $h=h_{0}+\sum_{i=n}^{k_{2}} h_{i} X^{i}$ be nonzero elements of $E\left[\Delta_{n}\right]$ with $h(0) \neq 0$ and let $g=\sum_{i=0}^{k_{3}} g_{i} X^{i} \in E[X] \backslash\{0\}$ with $g(0) \neq 0$ satisfying $\frac{g}{X^{m}}=\frac{f}{h} \in E\left[\Delta_{n}\right]_{S} \cap E\left[\Delta_{n}\right]_{X^{n} E[X]}$ for some nonnegative integer $m$. Then $X^{m} f=g h$; so $m=0$. By comparing coefficients of $f$ and $g h$, it is easy to see that $g_{i}=0$ for all $i=1, \ldots, n-1$. Hence $\frac{g}{X^{m}} \in E\left[\Delta_{n}\right]$. The reverse inclusion is clear. Thus $E\left[\Delta_{n}\right]$ is a weakly Krull domain.

(4) $\Rightarrow(5)$ By [Zafrullah 2003, Lemma 2.6], ht $T(X E[X])=\operatorname{dim}\left(E_{D \backslash\{0\}}[X]\right)$. By (4), ht $_{T_{n}}\left(X^{n} E[X]\right)=1$; so the comment before Remark 2.2 establishes that

$$
\operatorname{dim}\left(E_{D \backslash\{0\}}[X]\right)=1 .
$$

Thus $E_{D \backslash\{0\}}$ is a field. Also, since $X^{n} E[X]$ is a maximal $t$-ideal of $T_{n}, q f(D) \cap E=$ $D$ by Lemma 2.3(2). Finally, it follows directly from Theorem 1.3 that $E[X]$ is a weakly Krull domain.

(5) $\Rightarrow$ (2) [Anderson et al. 2006, Theorem 3.4].

(1) $\Rightarrow$ (2) In the proof of (2) $\Leftrightarrow(4)$, the integer $n \geq 2$ was arbitrary; so it suffices to show that $X^{F(\Gamma)+1} E[X]$ is a height-one maximal $t$-ideal of $T_{F(\Gamma)+1}$ and $E\left[\Delta_{F(\Gamma)+1}\right]$ is a weakly Krull domain. Assume that $R$ is a weakly Krull domain. Since $t-\operatorname{dim}(R)=1$ [Anderson et al. 1992, Lemma 2.1], $E\left[\Gamma^{*}\right]$ is a height-one maximal $t$-ideal of $R$ by Lemma 2.3(1); so $X^{F(\Gamma)+1} E[X]$ is a height-one maximal $t$-ideal of $T_{\Delta_{F(\Gamma)+1}}$ by Lemma 2.1 and the remark before Remark 2.2. Let $S_{1}=$ $\left\{X^{\alpha} \mid \alpha \in \Delta_{F(\Gamma)+1}\right\}$ and $S_{2}=\left\{X^{\alpha} \mid \alpha \in \Gamma\right\}$. Then $E\left[\Delta_{F(\Gamma)+1}\right]_{S_{1}}=R_{S_{2}}$ is a weakly Krull domain [Anderson et al. 1993, Proposition 4.7]. Also, $E\left[\Delta_{F(\Gamma)+1}\right]_{X^{F(\Gamma)+1} E[X]}$ is a weakly Krull domain because it is one-dimensional quasi-local. Note that $E\left[\Delta_{F(\Gamma)+1}\right]=E\left[\Delta_{F(\Gamma)+1}\right]_{S_{1}} \cap E\left[\Delta_{F(\Gamma)+1}\right]_{X^{F(\Gamma)+1} E[X]}$ as in the proof of (3) $\Rightarrow$ (4). Thus $E\left[\Delta_{F(\Gamma)+1}\right]$ is a weakly Krull domain.

Corollary 2.5. Let $R=D+E\left[\Gamma^{*}\right], T=D+X E[X], T_{n}=D+X^{n} E[X]$ and $\Delta_{n}=\{0\} \cup\left\{m \in \mathbb{N}_{0} \mid m \geq n\right\}$ for integers $n \geq 2$. If char $(E) \neq 0$, then the following statements are equivalent.

(1) $R$ is an AWFD.

(2) $R$ is a GWFD.

(3) $T$ is an AWFD. 
(4) $T$ is a GWFD.

(5) $T_{n}$ is an AWFD.

(6) $T_{n}$ is a GWFD.

(7) $X^{n} E[X]$ is a maximal $t$-ideal of $T_{n}, E\left[\Delta_{n}\right]$ is an AWFD and for each $0 \neq e \in E$, there exist an integer $m=m(e) \geq 1$ and $a$ unit $u$ of $E$ such that $u e^{m} \in D$.

(8) $X^{n} E[X]$ is a maximal $t$-ideal of $T_{n}, E\left[\Delta_{n}\right]$ is a GWFD and for each $0 \neq e \in E$, there exist an integer $m=m(e) \geq 1$ and $a$ unit $u$ of $E$ such that $u e^{m} \in D$.

(9) $q f(D) \cap E=D, E[X]$ is an AWFD and for each $0 \neq e \in E$, there exist an integer $m=m(e) \geq 1$ and $a$ unit $u$ of $E$ such that $u e^{m} \in D$.

(10) $q f(D) \cap E=D, E[X]$ is a GWFD and for each $0 \neq e \in E$, there exist an integer $m=m(e) \geq 1$ and $a$ unit $u$ of $E$ such that $u e^{m} \in D$.

Proof. (1) $\Rightarrow(2)$ and (5) $\Rightarrow$ (6) Their definitions lead to these implications.

(3) $\Leftrightarrow(9)$ [Anderson et al. 2006, Theorem 3.5].

(4) $\Leftrightarrow(10)$ [Anderson and Chang 2007, Corollary 2.10].

(7) $\Leftrightarrow(8)$ and (9) $\Leftrightarrow(10)$ See Corollary 1.5.

(7) $\Leftrightarrow(9)$ This equivalence follows from Corollary 1.5 and Lemma 2.3(2).

(3) $\Rightarrow$ (1) Assume that $T$ is an AWFD. Then $T$ is a weakly Krull domain [Anderson et al. 1992, Theorem 3.4]. Hence $E[X]$ is a weakly Krull domain by Theorem 2.4. Let $S=\left\{X^{m} \mid m \in \mathbb{N}_{0}\right\}$. Since $X$ is a prime element of $E[X], \mathrm{Cl}(E[X])=\mathrm{Cl}\left(T_{S}\right)$ is torsion [Anderson et al. 1993, Corollary 4.9]; so $E[X]$ is an AWFD [Anderson et al. 1992, Theorem 3.4]. Let $f \in R \backslash\{0\}$. Then there exists an integer $m \geq 1$ such that $f^{m}=X^{l} f_{1} \cdots f_{r}$ for some nonnegative positive integer $l$ and primary elements $f_{1}, \ldots, f_{r}$ of $E[X]$ with nonzero constant terms. Also, since $\operatorname{char}(E) \neq 0$, there exists an integer $k \geq F(\Gamma)+1$ such that $f_{i}^{k} \in E[\Gamma]$ for all $i=1, \ldots, r$; so $f^{m k}=X^{l k} f_{1}^{k} \ldots f_{r}^{k} \in E[\Gamma]$. Fix an $i \in\{1, \ldots, r\}$, and we claim that $\sqrt{f_{i}^{k} E[\Gamma]}$ is a prime ideal of $E[\Gamma]$ [Anderson et al. 2003b, Lemma 2.1]. Note that $\sqrt{f_{i} E[X]}=$ $\sqrt{f_{i}^{k} E[X]}$. If $\sqrt{f_{i}^{k} E[X]}=X E[X]$, then an easy calculation using a similar method as in the proof of $(2) \Rightarrow(1)$ in Theorem 2.4 shows that $\sqrt{f_{i}^{k} E[\Gamma]}=E\left[\Gamma^{*}\right]$ is a prime ideal. Assume that $\sqrt{f_{i}^{k} E[X]} \neq X E[X]$. Since $f_{i}(0) \neq 0, f_{i}^{k} E\left[X, X^{-1}\right]$ is a primary ideal of $E\left[X, X^{-1}\right]$; so $f_{i}^{k} E\left[X, X^{-1}\right] \cap E[\Gamma]$ is primary in $E[\Gamma]$. It is easy to see that $\sqrt{f_{i}^{k} E\left[X, X^{-1}\right]} \cap E[\Gamma]=\sqrt{f_{i}^{k} E[\Gamma]}$. Hence $\sqrt{f_{i}^{k} E[\Gamma]}$ is a prime ideal. Therefore we may assume that $f_{1}, \ldots, f_{r}$ are primary elements of $E[\Gamma]$ with nonzero constant terms and write $f^{m}=X^{l} f_{1} \cdots f_{r}$ as above. Note that for each $i=1, \ldots, r$, there exist a unit $u_{i}$ of $E$ and an integer $a_{i} \geq F(\Gamma)+1$ such that 
$u_{i} f_{i}(0)^{a_{i}} \in D$ as in the proof of (3) $\Leftrightarrow(9)$; so $u_{i} f_{i}^{a_{i}} \in R$. Let

$$
a=a_{1} \cdots a_{r}, \quad \hat{a_{i}}=\frac{a}{a_{i}}, \quad \text { and } \quad u=u_{1}^{\hat{a_{1}}} \cdots u_{r}^{\hat{a}_{r}} .
$$

Then $u f^{a m}=X^{a l}\left(u_{1} f_{1}^{a_{1}}\right)^{\hat{a}_{1}} \ldots\left(u_{r} f_{r}^{a_{r}}\right)^{\hat{a}_{r}}$ and $\sqrt{\left(u_{i} f_{i}^{a_{i}}\right)^{\hat{a}_{i}} E[\Gamma]}=\sqrt{f_{i} E[\Gamma]}$ for each $i=1, \ldots, r$. Since $t-\operatorname{dim}(E[\Gamma])=1,\left(u_{i} f_{i}^{a_{i}}\right)^{\hat{a}_{i}} E[\Gamma]$ is a primary ideal of $E[\Gamma]$ [Anderson et al. 2003b, Lemma 2.1] for each $1 \leq i \leq r$.

Claim. For each $1 \leq i \leq r,\left(u_{i} f_{i}^{a_{i}}\right)^{\hat{a}_{i}} R$ is a primary ideal of $R$.

Proof. Note that $\left(u_{i} f_{i}^{a_{i}}\right)^{\hat{a}_{i}} \in R$ and fix an $i \in\{1, \ldots, r\}$. We also note that $t$ $\operatorname{dim}(R)=1$ because $R$ is a weakly Krull domain by Theorem 2.4. Hence, by [Anderson et al. 2003b, Lemma 2.1], it suffices to show that $\sqrt{\left(u_{i} f_{i}^{a_{i}}\right)^{\hat{a}_{i}} R}$ is a prime ideal of $R$. If $\sqrt{\left(u_{i} f_{i}^{a_{i}}\right)^{\hat{a}_{i}} E[\Gamma]}=E\left[\Gamma^{*}\right]$, then it is easy to see that $\sqrt{\left(u_{i} f_{i}^{a_{i}}\right)^{\hat{a}_{i}} R}=$ $E\left[\Gamma^{*}\right]$ is a prime ideal of $R$. Assume that $\sqrt{\left(u_{i} f_{i}^{a_{i}}\right)^{\hat{a}_{i}} E[\Gamma]} \neq E\left[\Gamma^{*}\right]$. Then $\left(u_{i} f_{i}(0)^{a_{i}}\right)^{\hat{a}_{i}} \neq 0$. Now, we show that $\left(u_{i} f_{i}^{a_{i}}\right)^{\hat{a}_{i}} E\left[X, X^{-1}\right] \cap R=\left(u_{i} f_{i}^{a_{i}}\right)^{\hat{a}_{i}} R$. Let $h \in\left(u_{i} f_{i}^{a_{i}}\right)^{\hat{a}_{i}} E\left[X, X^{-1}\right] \cap R$. Note that we have

$$
\begin{aligned}
\left(u_{i} f_{i}^{a_{i}}\right)^{\hat{a}_{i}} E\left[X, X^{-1}\right] \cap R & \subseteq\left(u_{i} f_{i}^{a_{i}}\right)^{\hat{a}_{i}} E\left[X, X^{-1}\right] \cap E[\Gamma] \\
& =\left(u_{i} f_{i}^{a_{i}}\right)^{\hat{a}_{i}} E[\Gamma]
\end{aligned}
$$

by adapting the proof of $(2) \Rightarrow(1)$ in Theorem 2.4. So, we can write $h=\left(u_{i} f_{i}^{a_{i}}\right)^{\hat{a}_{i}} g$ for some $g \in E[\Gamma]$. Then

$$
g(0)=\frac{\left(u_{i} f_{i}(0)^{a_{i}}\right)^{\hat{a}_{i}}}{h(0)} \in q f(D) \cap E=D
$$

by Theorem 2.4; so $g \in R$. Therefore $h \in\left(u_{i} f_{i}^{a_{i}}\right)^{\hat{a}_{i}} R$, and hence

$$
\left(u_{i} f_{i}^{a_{i}}\right)^{\hat{a}_{i}} E\left[X, X^{-1}\right] \cap R \subseteq\left(u_{i} f_{i}^{a_{i}}\right)^{\hat{a}_{i}} R .
$$

The reverse inclusion is clear, and hence $\left(u_{i} f_{i}^{a_{i}}\right)^{\hat{a}_{i}} E\left[X, X^{-1}\right] \cap R=\left(u_{i} f_{i}^{a_{i}}\right)^{\hat{a}_{i}} R$. Since $\left(u_{i} f_{i}^{a_{i}}\right)^{\hat{a}_{i}} E[\Gamma]$ is a primary ideal of $E[\Gamma],\left(u_{i} f_{i}^{a_{i}}\right)^{\hat{a}_{i}} E\left[X, X^{-1}\right]$ is a primary ideal of $E\left[X, X^{-1}\right]$. Therefore $\sqrt{\left(u_{i} f_{i}^{a_{i}}\right)^{\hat{a}_{i}} R}=\sqrt{\left(u_{i} f_{i}^{a_{i}}\right)^{\hat{a}_{i}} E\left[X, X^{-1}\right]} \cap R$ is a prime ideal of $R$, and thus $\left(u_{i} f_{i}^{a_{i}}\right)^{\hat{a}_{i}} R$ is a primary ideal of $R$. The claim is proved.

If $l=0$, then $u f(0)^{a m}=\left(u_{1} f_{1}(0)^{a_{1}}\right)^{\hat{a}_{1}} \ldots\left(u_{r} f_{r}(0)^{a_{r}}\right)^{\hat{a}_{r}} \in D$; so $u$ is a unit of $D$ because $u$ is a unit of $E$. If $l \geq 1$, then $f^{a m}=u^{-1} X^{a l}\left(u_{1} f_{1}^{a_{1}}\right)^{\hat{a}_{1}} \cdots\left(u_{r} f_{r}^{a_{r}}\right)^{\hat{a}_{r}}$. Since $u^{-1} X^{a l} E[\Gamma]$ is a primary ideal of $E[\Gamma], u^{-1} X^{a l} R$ is a primary ideal of $R$ by imitating the previous proof. Hence $f^{a m}$ is a product of primary elements of $R$, and thus $R$ is an AWFD.

$(2) \Rightarrow(8)$ Assume that $R$ is a GWFD and fix an integer $n \geq 2$. Then $R$ is a weakly Krull domain [Anderson et al. 2003b, Corollary 2.3]; so $X^{n} E[X]$ is a height-one maximal $t$-ideal of $T_{n}$ by Theorem 2.4. 
Next, we claim that $E\left[\Delta_{n}\right]$ is a GWFD. Let $S_{1}=\left\{X^{m} \mid m \in \Delta_{n}\right\}$ and $S_{2}=$ $\left\{X^{m} \mid m \in \Gamma\right\}$. Then $E\left[\Delta_{n}\right]_{S_{1}}=E\left[X, X^{-1}\right]=R_{S_{2}}$ is a GWFD. Let $Q$ be a nonzero prime ideal of $E\left[\Delta_{n}\right]$. If $Q \cap S_{1} \neq \varnothing$, then $Q$ contains a primary element $X^{n}$ of $E\left[\Delta_{n}\right]$. If $Q \cap S_{1}=\varnothing$, then $Q E\left[\Delta_{n}\right]_{S_{1}}$ is a prime ideal of $E\left[\Delta_{n}\right]_{S_{1}}$; so $Q E\left[\Delta_{n}\right]_{S_{1}}$ contains a primary element $f \in E\left[X, X^{-1}\right]$. Note that $X$ is a unit of $E\left[X, X^{-1}\right]$ and $f^{k} \in E\left[\Delta_{n}\right]$ for some integer $k \geq 1$ because $\operatorname{char}(E) \neq 0$; so we may assume that $f \in E\left[\Delta_{n}\right]$ with $f(0) \neq 0$. Then

$$
f E\left[\Delta_{n}\right] \subseteq f E\left[\Delta_{n}\right]_{S_{1}} \cap E\left[\Delta_{n}\right] \subseteq Q E\left[\Delta_{n}\right]_{S_{1}} \cap E\left[\Delta_{n}\right]=Q
$$

so $Q$ contains a primary element $f$. Hence $E\left[\Delta_{n}\right]$ is a GWFD.

In order to check the final condition, let $e \in E \backslash\{0\}$. If $e$ is a unit of $E$, then we have nothing to prove. So, we assume that $e$ is not a unit of $E$ and let $h=e+X \in$ $E[X]$. Since $c(h)_{v}=E, h E[X]=h q f(E)[X] \cap E[X]$ [Anderson and Chang 2007, Lemma 2.1(1)]; so $h E[X]$ is a height-one prime ideal. Let $P=h E[X] \cap R$. Since $e$ is not a unit of $E, X^{F(\Gamma)+1} \notin P$; so $X^{\alpha} \notin P$ for all $\alpha \in \Gamma$. Therefore $h E\left[X, X^{-1}\right]=P R_{S_{2}} \subsetneq R_{S_{2}}$, and hence ht $R_{R}(P)=1$. Since $R$ is a GWFD, $P=\sqrt{g R}$ for some primary element $g \in R$ [Anderson et al. 2003b, Theorem 2.2]. Suppose to the contrary that $g(0)=0$. Since $E_{D \backslash\{0\}}$ is a field by Theorem $2.4, \frac{1}{e}=\frac{e^{\prime}}{d}$ for some $0 \neq d \in D$ and $e^{\prime} \in E$; so $e^{\prime} h=d+e^{\prime} X \in T$. Since $\operatorname{char}(E) \neq 0$, $\left(e^{\prime} h\right)^{k} \in h E[X] \cap R=P$ for some integer $k \geq 1$. Hence $\left(e^{\prime} h\right)^{k l} \in g R$ for some integer $l \geq 1$. However, this is impossible because $e \neq 0$. Therefore $g(0) \neq 0$. It is clear that $g R_{S_{2}}$ is a primary ideal of $R_{S_{2}}, g R_{S_{2}} \cap E[X]=g E[X], P R_{S_{2}}=\sqrt{g R_{S_{2}}}$ and $P R_{S_{2}} \cap E[X]=h E[X]$. Hence $g E[X]$ is a $h E[X]$-primary ideal. Therefore $g=u h^{m}$ for some $u \in q f(E)$ and some integer $m \geq 1$; so $u e^{m}=g(0) \in D$. Thus $u$ is a unit of $E$.

(3) $\Rightarrow(5)$ and $(6) \Rightarrow(8)$ These implications can be obtained by applying $\Gamma=\Delta_{n}$ to the proofs of (3) $\Rightarrow(1)$ and (2) $\Rightarrow(8)$, respectively.

We are closing this paper by showing that $R=D+E\left[\Gamma^{*}\right]$ is never a WFD and the assumption "char $(E)=0$ " is essential in Corollary 2.5.

Remark 2.6. Assume that $R=D+E\left[\Gamma^{*}\right]$ is a WFD or an AWFD. Let $h=1+X \in$ $E[X], P=h E[X] \cap R$ and let $M$ be a maximal $t$-ideal of $R$. If $M=E\left[\Gamma^{*}\right]$, then $P R_{M}=R_{M}$ because $1+(-1)^{F(\Gamma)} X^{F(\Gamma)+1} \in P \backslash E\left[\Gamma^{*}\right]$. Assume that $M \neq E\left[\Gamma^{*}\right]$. Since $c(h)_{v}=E, h q f(E)[X] \cap E[X]=h E[X]$ [Anderson and Chang 2007, Lemma 2.1(1)]. Let $S=\left\{X^{m} \mid m \in \Gamma\right\}$. Then $P E\left[X, X^{-1}\right]=h E\left[X, X^{-1}\right]$; so $P R_{M}=h R_{M}$ is principal. Hence $P$ is $t$-locally principal, and thus $P$ is $t$-invertible [Anderson et al. 1992, Lemma 2.2].

(1) If $R$ is a WFD, then $P=g R$ for some $g \in R$ with $g(0) \neq 0$ [Anderson and Zafrullah 1990, Theorem $]$. Note that $h E\left[X, X^{-1}\right]=g E\left[X, X^{-1}\right]$; so $g=u h$ for some unit $u$ of $E$. Hence $u h \in R$, which is impossible. Thus $R$ is not a WFD. 
(2) Assume that $R$ is an AWFD. Then $P^{m}=g R$ for some integer $m \geq 1$ and $g \in R$ with $g(0) \neq 0$ [Anderson et al. 1992, Theorem 3.4]. We note that

$$
h^{m} E\left[X, X^{-1}\right]=g E\left[X, X^{-1}\right] ;
$$

so $u h^{m}=g$ for some unit $u$ of $E$. Hence $u h^{m} \in R$. However, this can not happen if $\operatorname{char}(E)=0$. Thus $R$ is never an AWFD whenever $\operatorname{char}(E)=0$.

\section{Acknowledgements}

The author would like to thank the referee for several valuable comments and suggestions which resulted in an improved version of the paper. Also, the author is deeply grateful to professor Gyu Whan Chang for pointing out Remark 1.6(1).

This work was supported by the Brain Korea 21 Project Team to Nurture the Next Generation of First-class Mathematical Scientists by the Korean Government.

\section{References}

[Anderson and Chang 2004] D. F. Anderson and G. W. Chang, "The class group of $D[\Gamma]$ for $D$ an integral domain and $\Gamma$ a numerical semigroup", Comm. Algebra 32:2 (2004), 787-792. MR 2005g:13021 Zbl 1092.13017

[Anderson and Chang 2005] D. F. Anderson and G. W. Chang, "Homogeneous splitting sets of a graded integral domain", J. Algebra 288:2 (2005), 527-544. MR 2006g:13001 Zbl 1084.13001

[Anderson and Chang 2007] D. F. Anderson and G. W. Chang, "Almost splitting sets in integral domains. II”, J. Pure Appl. Algebra 208:1 (2007), 351-359. MR 2007i:13004 Zbl 1171.13300

[Anderson and Zafrullah 1990] D. D. Anderson and M. Zafrullah, "Weakly factorial domains and groups of divisibility”, Proc. Amer. Math. Soc. 109:4 (1990), 907-913. MR 90k:13015 Zbl 0704. 13008

[Anderson et al. 1992] D. D. Anderson, J. L. Mott, and M. Zafrullah, "Finite character representations for integral domains", Boll. Un. Mat. Ital. B (7) 6:3 (1992), 613-630. MR 93k:13001 Zbl 0773.13004

[Anderson et al. 1993] D. D. Anderson, E. G. Houston, and M. Zafrullah, " $t$-linked extensions, the $t$-class group, and Nagata's theorem", J. Pure Appl. Algebra 86:2 (1993), 109-124. MR 94e:13036 Zbl 0777.13002

[Anderson et al. 1995] D. D. Anderson, D. F. Anderson, and M. Zafrullah, "A generalization of unique factorization", Boll. Un. Mat. Ital. A (7) 9:2 (1995), 401-413. MR 96d:13027 Zbl 0919. 13001

[Anderson et al. 2003a] D. F. Anderson, G. W. Chang, and J. Park, " $D\left[X^{2}, X^{3}\right]$ over an integral domain D", pp. 1-14 in Commutative ring theory and applications (Fez, 2001), Lecture Notes in Pure and Appl. Math. 231, Dekker, New York, 2003. MR 2004k:13011 Zbl 1080.13510

[Anderson et al. 2003b] D. F. Anderson, G. W. Chang, and J. Park, "Generalized weakly factorial domains”, Houston J. Math. 29:1 (2003), 1-13. MR 2004e:13003 Zbl 1029.13012

[Anderson et al. 2006] D. F. Anderson, G. W. Chang, and J. Park, "Weakly Krull and related domains of the form $D+M, A+X B[X]$ and $A+X^{2} B[X]$ ", Rocky Mountain J. Math. 36:1 (2006), 1-22. MR 2007h:13027 Zbl 1133.13022 
[Chang 2005] G. W. Chang, "Almost splitting sets in integral domains", J. Pure Appl. Algebra 197:13 (2005), 279-292. MR 2005j:13005 Zbl 1091.13001

[Chang et al. 2012] G. W. Chang, H. Kim, and J. W. Lim, "Numerical semigroup rings and almost Prüfer $v$-multiplication domains", preprint, 2012. Accepted in Comm. Algebra.

[El Baghdadi et al. 2002] S. El Baghdadi, L. Izelgue, and S. Kabbaj, "On the class group of a graded domain”, J. Pure Appl. Algebra 171:2-3 (2002), 171-184. MR 2003d:13012 Zbl 1058.13006

[Gilmer 1992] R. Gilmer, Multiplicative ideal theory, Queen's Papers in Pure and Applied Mathematics 90, Queen's University, Kingston, ON, 1992. MR 93j:13001 Zbl 0804.13001

[Gilmer and Martin 1990] R. Gilmer and M. B. Martin, "On the Picard group of a class of nonseminormal domains”, Comm. Algebra 18:10 (1990), 3263-3293. MR 91g:13017

[Kang 1989] B. G. Kang, "Prüfer $v$-multiplication domains and the ring $R[X]_{N_{v}}$ ", J. Algebra 123:1 (1989), 151-170. MR 90e:13017 Zbl 0668.13002

[Kaplansky 1970] I. Kaplansky, Commutative rings, Allyn and Bacon, Boston, 1970. Reprinted Polygonal Publishing House, Washington, 1994.

[Li 2012] Q. Li, “On almost Prüfer v-multiplication domains”, Algebra Colloq. (2012).

[Lim 2012] J. W. Lim, "Generalized Krull domains and the composite semigroup ring $D+E\left[\Gamma^{*}\right]$ ”, J. Algebra 357 (2012), 20-25.

[Zafrullah 2003] M. Zafrullah, "Various facets of rings between $D[X]$ and $K[X]$ ", Comm. Algebra 31:5 (2003), 2497-2540. MR 2004d:13029 Zbl 1052.13003

Received September 17, 2011. Revised April 10, 2012.

JUNG WOOK LIM

DEPARTMENT OF MATHEMATICS

SOGANG UNIVERSITY

SEOUL 121-742

SOUTH KoreA

lovemath@postech.ac.kr

jwlim@sogang.ac.kr 


\title{
PACIFIC JOURNAL OF MATHEMATICS
}

\author{
http://pacificmath.org \\ Founded in 1951 by \\ E. F. Beckenbach (1906-1982) and F. Wolf (1904-1989)
}

\section{EDITORS}

V. S. Varadarajan (Managing Editor)

Department of Mathematics

University of California

Los Angeles, CA 90095-1555

pacific@math.ucla.edu

Vyjayanthi Chari

Department of Mathematics

University of California

Riverside, CA 92521-0135

chari@math.ucr.edu

\section{Robert Finn}

Department of Mathematics Stanford University

Stanford, CA 94305-2125

finn@math.stanford.edu

Kefeng Liu

Department of Mathematics

University of California

Los Angeles, CA 90095-1555

liu@math.ucla.edu
Darren Long

Department of Mathematics

University of California

Santa Barbara, CA 93106-3080

long@math.ucsb.edu

Jiang-Hua Lu

Department of Mathematics

The University of Hong Kong

Pokfulam Rd., Hong Kong jhlu@maths.hku.hk

Alexander Merkurjev

Department of Mathematics

University of California

Los Angeles, CA 90095-1555

merkurev@math.ucla.edu
Sorin Popa

Department of Mathematics University of California

Los Angeles, CA 90095-1555 popa@math.ucla.edu

Jie Qing

Department of Mathematics

University of California

Santa Cruz, CA 95064

qing@cats.ucsc.edu

Jonathan Rogawski

Department of Mathematics

University of California

Los Angeles, CA 90095-1555

jonr@math.ucla.edu

\section{PRODUCTION}

pacific@math.berkeley.edu

\section{SUPPORTING INSTITUTIONS}

ACADEMIA SINICA, TAIPEI

CALIFORNIA INST. OF TECHNOLOGY INST. DE MATEMÁTICA PURA E APLICADA KEIO UNIVERSITY

MATH. SCIENCES RESEARCH INSTITUTE NEW MEXICO STATE UNIV.

OREGON STATE UNIV.

\author{
STANFORD UNIVERSITY \\ UNIV. OF BRITISH COLUMBIA \\ UNIV. OF CALIFORNIA, BERKELEY \\ UNIV. OF CALIFORNIA, DAVIS \\ UNIV. OF CALIFORNIA, LOS ANGELES \\ UNIV. OF CALIFORNIA, RIVERSIDE \\ UNIV. OF CALIFORNIA, SAN DIEGO \\ UNIV. OF CALIF., SANTA BARBARA
}

\author{
UNIV. OF CALIF., SANTA CRUZ \\ UNIV. OF MONTANA \\ UNIV. OF OREGON \\ UNIV. OF SOUTHERN CALIFORNIA \\ UNIV. OF UTAH \\ UNIV. OF WASHINGTON \\ WASHINGTON STATE UNIVERSITY
}

These supporting institutions contribute to the cost of publication of this Journal, but they are not owners or publishers and have no responsibility for its contents or policies.

See inside back cover or pacificmath.org for submission instructions.

The subscription price for 2012 is US \$420/year for the electronic version, and \$485/year for print and electronic.

Subscriptions, requests for back issues from the last three years and changes of subscribers address should be sent to Pacific Journal of Mathematics, P.O. Box 4163, Berkeley, CA 94704-0163, U.S.A. Prior back issues are obtainable from Periodicals Service Company, 11 Main Street, Germantown, NY 12526-5635. The Pacific Journal of Mathematics is indexed by Mathematical Reviews, Zentralblatt MATH, PASCAL CNRS Index, Referativnyi Zhurnal, Current Mathematical Publications and the Science Citation Index.

The Pacific Journal of Mathematics (ISSN 0030-8730) at the University of California, c/o Department of Mathematics, 969 Evans Hall, Berkeley, CA 94720-3840, is published monthly except July and August. Periodical rate postage paid at Berkeley, CA 94704, and additional mailing offices. POSTMASTER: send address changes to Pacific Journal of Mathematics, P.O. Box 4163, Berkeley, CA 94704-0163.

PJM peer review and production are managed by EditFLOW ${ }^{\mathrm{TM}}$ from Mathematical Sciences Publishers.

PUBLISHED BY PACIFIC JOURNAL OF MATHEMATICS

at the University of California, Berkeley 94720-3840

A NON-PROFIT CORPORATION

Typeset in LATEX

Copyright $(02012$ by Pacific Journal of Mathematics 


\section{PACIFIC JOURNAL OF MATHEMATICS}

Volume $257 \quad$ No. $1 \quad$ May 2012

Energy and volume of vector fields on spherical domains

FABiano G. B. BRito, ANDRÉ O. Gomes and Giovanni S. Nunes

Maps on 3-manifolds given by surgery

BOLDIZSÁR KALMÁR and ANDRÁS I. STIPSICZ

Strong solutions to the compressible liquid crystal system

Yu-Ming ChU, Xian-Gao LiU and XIAO LIU

Presentations for the higher-dimensional Thompson groups $n V$

Johanna HenNig and FranCESCo MatuCCI

Resonant solutions and turning points in an elliptic problem with oscillatory

boundary conditions

ALFONSO CASTRO and ROSA PARDO

Relative measure homology and continuous bounded cohomology of topological pairs

\section{Roberto Frigerio and CRISTINA PAgLiAnTINi}

Normal enveloping algebras

ALEXANDRE N. GrishKov, Marina RASSKazova and SALVATORE SICILIANO

Bounded and unbounded capillary surfaces in a cusp domain

YASUNORI AOKI and DAVID SIEGEL

On orthogonal polynomials with respect to certain discrete Sobolev inner product

Francisco Marcellán, Ramadan Zejnullahu, Bujar Fejzullahu and EDMUNDO HUERTAS

Green versus Lempert functions: A minimal example

PASCAL THOMAS

Differential Harnack inequalities for nonlinear heat equations with potentials under the Ricci flow

JIA-YONG WU

On overtwisted, right-veering open books

PAOLO LISCA

Weakly Krull domains and the composite numerical semigroup ring $D+E\left[\Gamma^{*}\right]$

JUNG WOOK LIM

Arithmeticity of complex hyperbolic triangle groups 